\title{
Innately Adaptive Robotics through Embodied Evolution
}

\author{
Siavash Haroun Mahdavi and Peter J. Bentley \\ Department of Computer Science \\ University College London \\ Gower St. London WC1E 6BT, UK \\ \{mahdavi, p.bentley\}@cs.ucl.ac.uk
}

\begin{abstract}
Autonomous adaptation in robots has become recognised as crucial for devices deployed in remote or inhospitable environments. The aim of this work is to investigate autonomous robot adaptation, focussing on damage recovery and adaptation to unknown environments. An embodied evolutionary algorithm is introduced and its capabilities demonstrated with experimental results. This algorithm is shown to be able to control the motion of a robot snake effectively; this same algorithm inherently recovers the snake's motion after damage. Another experiment shows that the algorithm is capable of contorting a shape-changing antenna in such a way as to minimise the affect of background noise on it, thus allowing the antenna to achieve a better signal.
\end{abstract}

\section{Introduction}

Modelling the world with any accuracy is difficult. Modelling how an autonomous robot will react on another world is virtually impossible. Robot controllers that rely on such modelling are brittle and susceptible to catastrophic failure. The goal of autonomous, self-sustaining robots will not be reached by predicting every eventuality and designing solutions for every problem. The complexity of interactions between robot and environment mean that there will always be an unforeseen problem that results in mission failure. Instead, a better way to achieve long-term survival of robots is to make robots innately adaptive. Every part of their form and function must enable them to change, to alter themselves. They must learn how to move themselves in unknown environments, whether damaged or not. They must adapt to their environment and increase their ability to transmit or receive signals amongst themselves. They must be embodied in their environments.

This work outlines a method of control that is embodied in two physical robots: a self-adaptive snake robot and an adaptive antenna. Both use shape memory alloy for actuation. Here we investigate the use of an evolutionary algorithm to evolve controllers in these physical systems, under different environmental conditions. Embodied evolution creates and tests controllers in these physical devices, in real environments, giving them an innate adaptability. This adaptability is assessed by repeatedly damaging the robot snake and measuring the degree of recovery, and by placing the antenna in a challenging environment and measuring its ability to adapt and receive a radio signal.

\section{Background}

The work described here makes use of and is impacted by several related areas of research. The following section summarises relevant work in the field of robotics, antennas and control, focussing on self-repairing, evolutionary and smart-material-based robots, evolutionary antennas, and embodied evolutionary control. 


\subsection{Robotics}

\subsubsection{Self-Repairing Robots}

Although evolutionary design [1] is common, little work is evident in the field of self-repairing robotics. Most current research seems to be limited to theory and future predictions. Bererton and Khosla propose that there will be large self-sufficient robot colonies operating on distant planets. They describe methods where teams of robots could repair each other, and they have done experiments on visual docking systems for this purpose [3]. Michael claims that in the future 'fractal robots' will emerge which can completely change their shape and so if damaged, can reassemble them selves to recover their previous capabilities [26]. Kamimura has built robots that can reconfigure themselves to perform different tasks (as have Dittrich et al [6]), though he has not yet looked into the idea of self-repair [19]. Perhaps the work most similar to that described here was performed by Støy [31], in which a chain robot made of nine modules is robust to signal loss and able to continue effective locomotion. However, unlike the system described here, the controllers are preprogrammed and incapable of learning novel movement strategies after damage to the robot. More recently, Huntsberger et al [15] presented an algorithm for onboard adaptive learning of weights within a rover hierarchical behavior control framework called SMART. Results indicated that an additional $52.9 \%$ of missions would potentially be successful with adaptive learning.

\subsubsection{Evolutionary Robotics}

The field of adaptive robotics is not a new one. Many robots that use standard methods of movement like motors and pistons have been evolved from the design stage and all the way through to the control stage [1]. For a thorough survey, readers are advised to read [16] and [25]. Further examples include Jakobi, who endeavoured to build a simulation within which control systems for real robots could evolve [18]. Hornby evolved L-Systems to generate complicated multi-component virtual creatures [14]. Husbands used evolutionary robotics techniques to develop control networks and visual morphologies to enable a robot to achieve target discrimination tasks under very noisy lighting conditions [17]. Mihalachi and Munerato created a robot snake able to move like an earthworm [27][28].

\subsubsection{Smart Materials in Robotics}

The use of smart materials in robotics has been investigated previously. For example, Kárník looked at the possible applications of walking robots, which use artificial muscles with smart materials as a drive [20]. Mills's book, aimed at beginners, teaches how to make simple eight-legged robots using smart materials as actuators [29]. However, no one has used smart materials and evolutionary algorithms together in a robot before. This work uses nitinol wires as muscles within a robot snake and adaptive antenna, with the muscle activation sequences evolved using genetic algorithms and finite state machines.

Nitinol, an alloy made of Nickel and Titanium, was developed by the Naval Ordinance Laboratory. When current runs through it, thus heating it to its activation temperature, it changes shape to the shape that it has been 'trained' to remember. The wires used in this project simply reduce in length, (conserving their volume and thus getting thicker), by about 5-8 \%. Shape Memory Alloys, when cooled from the stronger, high temperature form (Austenite), undergo a phase transformation in their crystal structure to the weaker, low temperature form (Martensite). This phase transformation allows these alloys to be super elastic and have shape memory [13]. The phase transformation occurs over a narrow range of temperatures, although the beginning and end of the transformation actually spread over a much larger range of temperatures. Hysteresis occurs, as the temperature curves do not overlap during heating and cooling, see Fig. 1 [13]. With thick wires, this could bring about problems for our robot as the SMA wires would take some time before returning to their original lengths, however, due to the very small diameter of the SMA wires used $(\sim 0.15 \mathrm{~mm})$, the hysteresis is almost negligible as the cooling to below the Martensite temperature, (Mf), is almost instantaneous [9]. 


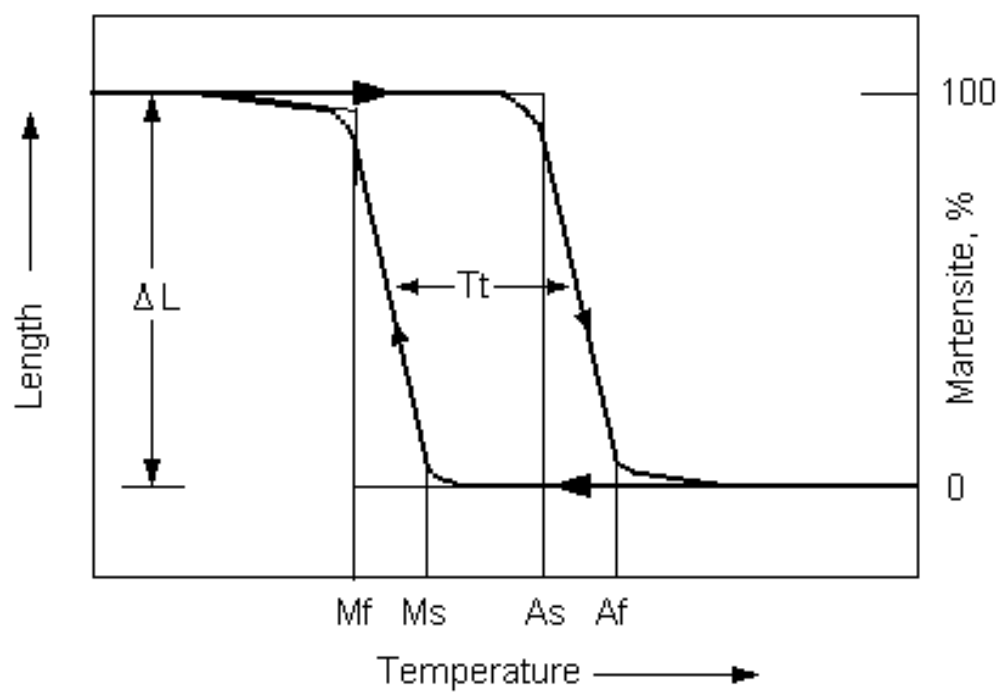

Fig. 1. Graph showing change in length during heating and cooling. The hysteresis is represented by Tt.

\subsection{Antennas}

\subsubsection{Antenna Functions}

An antenna is a device that captures radio-frequency signals. It can be any conductive structure that can carry an electric current. Antennas can be transmitters or receivers. Transmitter antennas carry a time varying electrical current and radiate an electromagnetic wave. Receiver antennas do exactly the opposite. They pick electromagnetic waves and convert them into an electrical current. A passive antenna, that is one with no amplifiers attached, will have the same characteristics whether it is transmitting or receiving. [7]

The antenna being evolved in this work is a receiver antenna. Using up to 16 SMA wires, it will attempt to maximize the signal strength being received by changing its shape.

\subsubsection{Evolutionary Antennas}

There has been much work on evolutionary design [2] and research has begun on using shape memory alloys with computers [9][10][11]. Most previous work related to antennas concentrates on the use of evolutionary algorithms in the design and optimisation of antenna structures. This is very different from the work proposed here that seeks to, given a general shape, vary the shape of the antenna intelligently to adapt to real world situations.

Linden was the first to use evolutionary algorithms to design antenna structures [21]. His work included the optimising of Yagi-Uda antenna structures, where the parallel wires of the Yagi-Udi structure were rotated about the central boom, as specified by a genetic algorithm. [21]. He went on to evolve designs of crooked-wire antenna (a single wire bent several times into a specific configuration), and treelike genetic antennas [2].

At NASA Ames Research Center, Lohn et al have used evolutionary algorithms to determine the size and spacing of the elements within a Yagi-Uda antenna [22]. More recently they have used a co-evolutionary algorithm to optimize the design parameters of a quadrifilar helical antenna [23]. Their plans include work to create antenna for space probes that will be designed to cope with the noise generated by other systems on the device. 
Finally, the surprising work of Bird and Layzell [4] has also demonstrated the evolution of an antenna design, albeit by accident. In their experiment to evolve an electronic circuit that produced an oscillating signal as output, they discovered that instead, evolution had produced a primitive receiver that was receiving the background noise created by a nearby computer monitor and modifying it.

\subsection{Robot Control}

\subsubsection{Embodied Control}

Embodied control of robots is the creation of adaptive control mechanisms that learn and adapt within physical devices that are deployed in real environments (in contrast to control mechanisms that learn and adapt off-line in simulators). Embodiment is becoming increasing important to the field of robotics. Tom Quick [30] gives a minimal and precise definition as follows, see Fig. 2:

A system $\mathrm{X}$ is embodied in an environment $\mathrm{E}$ if perturbatory channels exist between the two. That is, $\mathrm{X}$ is embodied in $\mathrm{E}$ if for every time $t$ at which $\mathrm{X}$ and $\mathrm{E}$ exist, some subset of $\mathrm{E}$ 's possible states have the capacity to perturb X's state, and some subset X's possible states have the capacity to perturb E's state. [30]

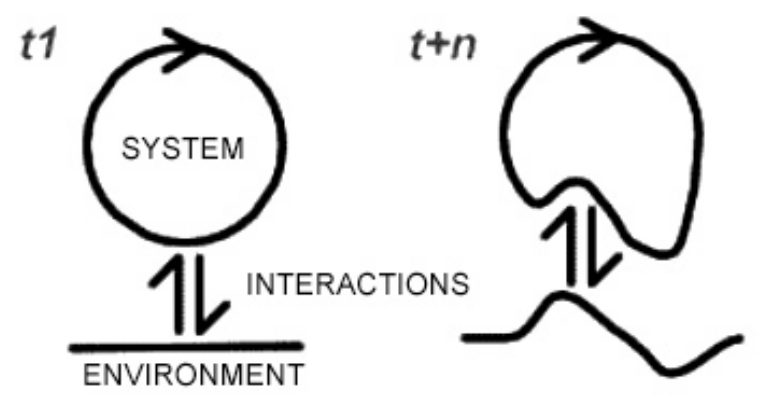

Fig.2 An embodied system interacting with its environment.

As shown in figure 1, over time an embodied system will have some impact on its environment, and its environment will have some impact on the system. Where such mutual influence results in adaptation of form or functionality, the system is said to be structurally coupled to its environment [30].

\subsubsection{Embodied Evolutionary algorithms}

As is evident in the above literature, many robot controllers have been evolved with the use of evolutionary algorithms. To be embodied means to affect and be affected by your environment. Robots whose controllers have been evolved within a simulator often have problems of transference and do not demonstrate the ability to adapt to changes within the real environment [5][16]. The work in this paper proposes that by evolving the controller in situ, in the real environment, a controller is created that is both effective and inherently adaptive. This is embodied according to the definition above, as the controller and robot interacts with and adapts to a real, physical environment and the environment interacts and affects the robot and its controller [30][8]. This aspect of embodied evolutionary algorithms is extremely useful in situations where the robot in question is no longer at arms reach. Once the robot has been deployed, it is critical that it performs its tasks well. Later results described in this paper will show that embodied evolutionary algorithms allow the robot to adapt to the conditions and changes in the environment. These conditions could very well be ones that were previously unpredicted by the designer back on Earth. The results will also show that since the controller is constantly adapting to the environment, that the robot and the adaptive antenna will be able to adapt to changes in the environment whether they be damage to the robot itself or varying noise conditions for the adaptive antenna. 


\section{Self-adapting Snake (SAS)}

The notion of adaptation through embodied evolutionary control is investigated in two devices: a "snake" robot and a shape-changing antenna. This section focuses on the self-adapting snake.

\subsection{Snake Robot Prototype}

The robot employs shape memory alloys (SMAs) as actuators, with the body constructed from wood and foam (supplying a restoring force for the shape memory alloys). Experiments are described that illustrate how a simple genetic algorithm is able to effectively control the motion of the robot snake without any previous domain knowledge about how the SMAs behave, the structure of the robot or the surface upon which it is travelling. All of this is possible, it is argued, because the genetic algorithm is embodied within its environment.

\subsubsection{Body}

The self-adapting snake (SAS) body went through several designs before reaching the final design. The main criterion was that it provided a restoring force great enough to restore the wires to their original lengths after each activation. Foam was chosen for the job for all but one of the snake designs where plastic wire insulators were used [9].

The SAS used in the experiments used four SMA wires (diameter $=1.5 \mathrm{~mm}$, activation (Austenite) temperature $=70^{\circ} \mathrm{C}$, recommended current $200 \mathrm{~mA}$ ), that were connected all together at one side with a nut and bolt, which in turn was connected to a piece of normal wire. This wire ran through the middle of the snake and supplied the power, much like a spinal chord carries nerves impulses to muscles through the body, see Fig. 3. The total weight of the SAS was approximately $50 \mathrm{~g}$.

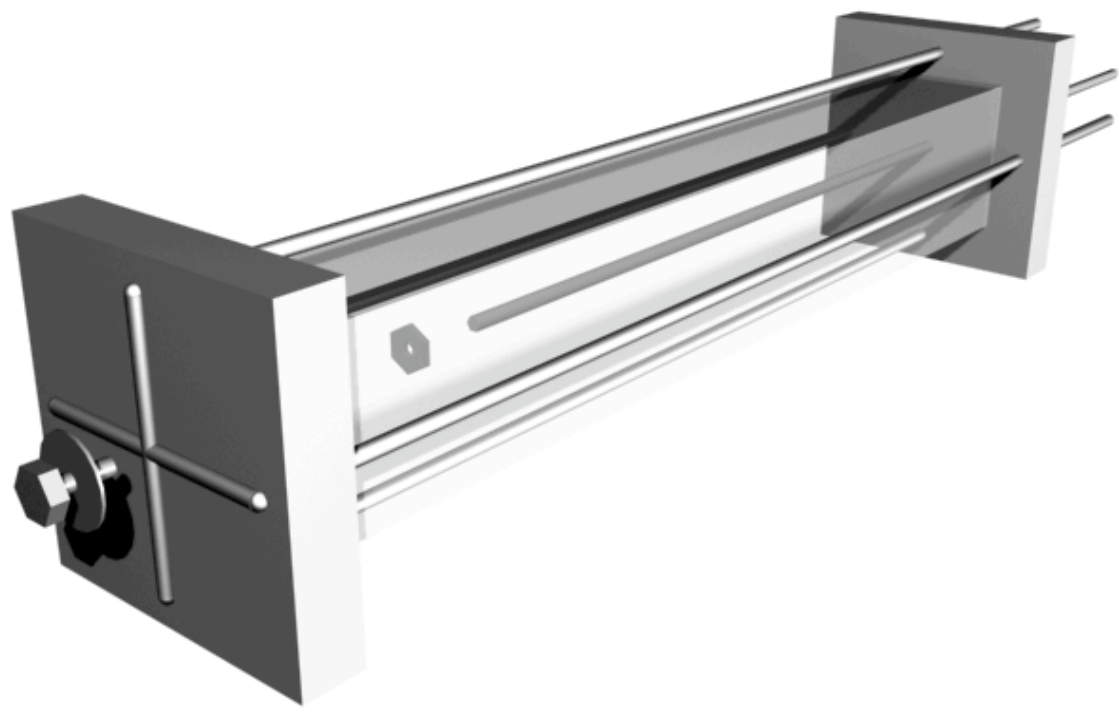

Fig. 3. Four wires were arranged at the top, bottom, left and right of the SAS.

\subsubsection{Brain}

A finite state machine was used to determine the wire activation sequences; these were then evolved using a standard single-point crossover, elitist genetic algorithm with roulette wheel selection. Elitism in this GA works simply by taking the $\mathrm{n}(\mathrm{n}=2$ for the experiment) best members of the population and in addition to using them 
to generate members of the next generation, they are also placed directly into the next generation. In doing such, they naturally ensure that the maximum distance travelled during the coming generations never decreases, and they are also given another chance to influence the next population if they still have the highest fitness.

Each member of the population was made up of a sting of 0 s and 1s. Each string consisted of two segments, 'sequence' and 'next time', for a simple example see Fig. 4. The 'sequence' was the part that was sent to the SAS, and determined which wires were to be activated at that particular time. The 'next time' was the part that told the program to which time slot in the current row it should then jump. The recommended technique for activating the SMA wires is to pulse them. This is because prolonged heating causes 'hotspots' to occur and these damage the wire and reduce its life span. Therefore, each sequence is in fact pulsed 7 times before moving on to the next sequence [9].
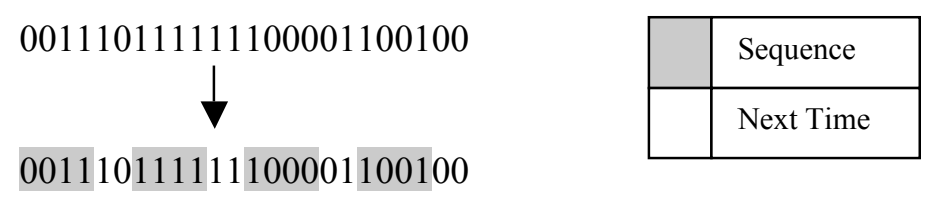

Fig. 4. Example string using 24 instead of 640 bits. Binary string is split into 'sequence' and 'next time' segments.

For the SAS, the 'sequence' length was four bits, and the 'next time' length was six bits, therefore the total length of each string was $2^{6} \times(4+6)=640$ bits.

The program was allowed to send 20 sequences to the SAS before stopping, each lasting a total of approximately 40 seconds. Finite state machines that acted as repeating patterns were easily created if the jump points pointed to each other in a loop of some sort. Indeed, very rarely was there a string that didn't loop at all.

The computer interfacing hardware, though quite complex, had two simple tasks to perform. The first was to supply enough power to each SMA wire $(\sim 200 \mathrm{~mA})$. This was achieved with the use of some Darlington amplifiers. The second task to perform was the ability to activate muscles in parallel. For this, the microcontroller used was the Motorola $\mathrm{MC} 68 \mathrm{H}(\mathrm{R}) \mathrm{C} 908 \mathrm{JK} 1$. It had 20 pins and with the structure of the circuit board was capable of activating up to 12 pins in parallel (PTBx and PTDx excluding PTB0 and PTB3) [24].

\subsubsection{Experimental Setup}

The SAS was connected to the microcontroller circuit board using a long lead ( $600 \mathrm{~mm})$ so that it did not have to pull much weight when trying to move. The SAS was placed on grid paper and a starting point was marked out. As the program started, an initial population of randomly generated finite state machines was created. Each individual finite state machine would then be sent to the SAS for evaluation. The fitness of any member was determined solely on how far the snake travelled (in $\mathrm{mm}$ ) in the forward direction [9].

The final configuration consisted of the SAS being placed on top of a platform about $500 \mathrm{~mm}$ above the level of the circuit board and power supply. The wires were then draped over the edge of the platform. As the SAS moved forward, the wires were pushed down over the edge and so out of the way of the snake. There was concern over whether the weight of the wires was assisting in pulling the SAS along but much experimentation with the SAS ensured that the wires were pushed along and did not pull. The important factor was that the resistance of the wires was more or less constant no matter how far the SAS moved [9]. Following this, an experiment was performed to investigate the evolution of movement for the SAS. The SAS's motion was evolved for 25 generations and the corresponding fitnesses were stored. 


\subsubsection{Results}

Unfortunately the experiment had to be temporarily halted after only 8 generations. This was because one of the SMA wires snapped.

The SMA wire snapped at exactly the corner of the hole that it was fed through. It is believed that it snapped due to the sharp corner that it rubbed against and not because of overheating [9]. This would normally spell the end for any other robot, however one of the hypotheses of this research is that such techniques enable selfadaptation through embodied evolution and so it seemed only logical that if the SAS was truly self-adapting that it should be able to adapt to the loss of a muscle and learn other ways to move.

To test this, evolution was continued from the point at which the SMA wire snapped (beginning of $8^{\text {th }}$ generation). It seems likely that every member of the population used the $4^{\text {th }}$ wire to move at some point during its sequence and so the fitness of the whole populations dropped from an average distance of $13.25 \mathrm{~mm}$ to an average of only $1.95 \mathrm{~mm}$, see fig 6 . The members that did slightly better than the rest were probably members that had moved quite proficiently and had also used less of wire four. So these sequences were propagated more and more in the following generations. It took around 10 generations for any sequence to be found that moved more than $6 \mathrm{~mm}$. Then came a sequence that simply alternated between the top and bottom wire. This caused the SAS to move over $20 \mathrm{~mm}$. Since elitist selection was being implemented, this sequence was not lost and variations spread throughout the proceeding generations increasing the fitness of the whole population considerably, see Fig. 5.

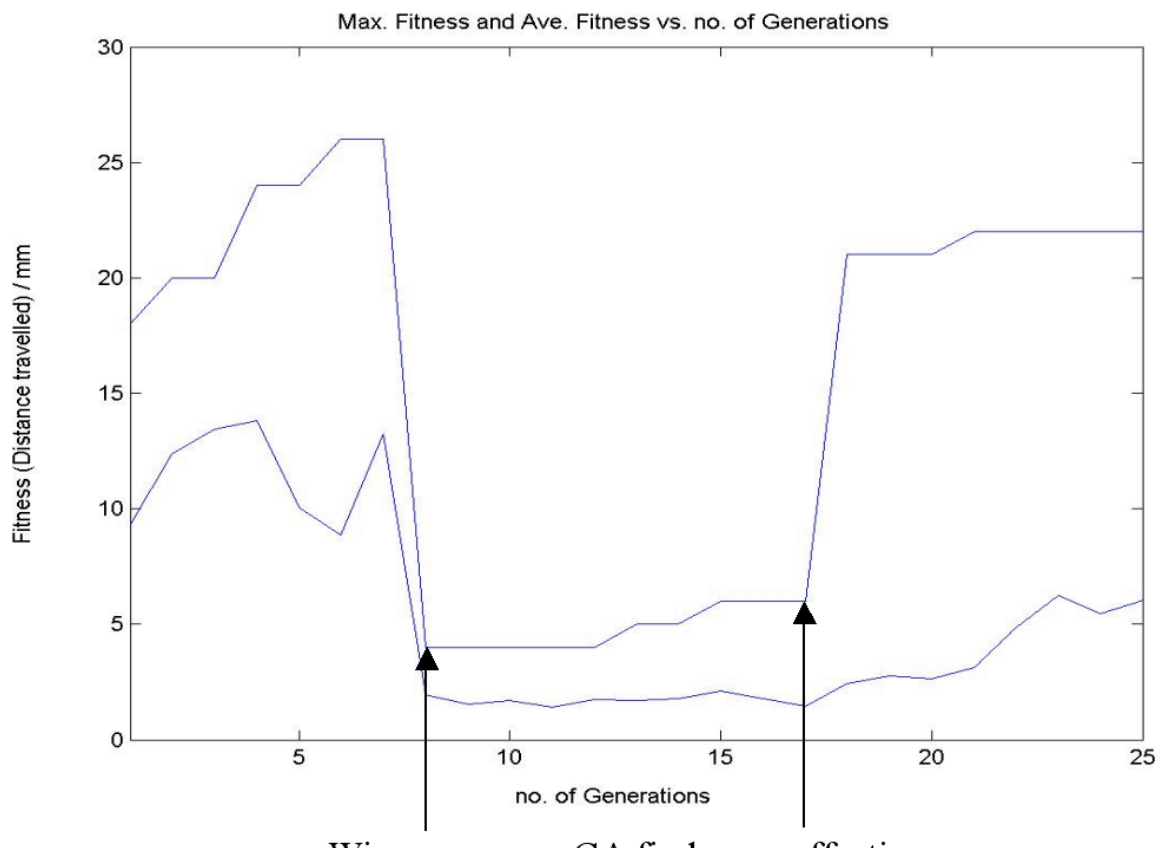

Wire snaps GA finds new effective sequence

Fig. 5. Maximum fitness and average fitness plotted at each generation.

\subsubsection{Analysis}

During the evolution of the SAS, numerous interesting methods of locomotion were carried out. Though most of them were unsuccessful, this section seeks to analyse the physics behind two of the more efficient evolved sequences.

Though the sequence lengths could vary from a length of one to a maximum of sixty-four, the sequences that travelled the furthest seemed to have short repetitive loops. 

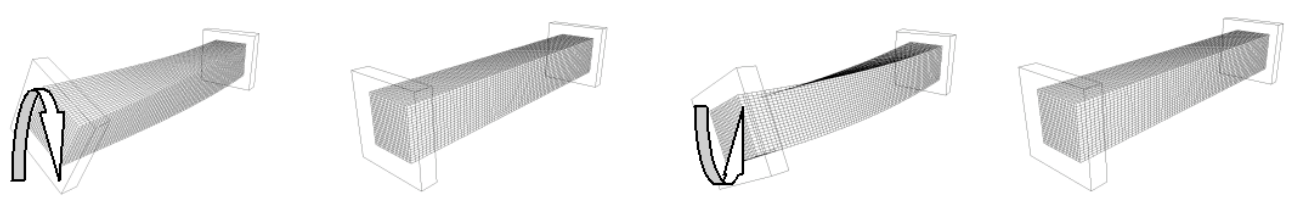

\begin{tabular}{|c|c|c|c|}
\hline TOP & TOP & TOP & TOP \\
LEFT RIGHT & LEFT RIGHT & LEFT RIGHT & LEFT RIGHT \\
BOTTOM & BOTTOM & BOTTOM & BOTTOM \\
\hline $0 \longrightarrow 1 \longrightarrow 2 \longrightarrow 3$ \\
\hline
\end{tabular}

Fig. 6. Activation sequence of best sequence found resembles cobra undulations.

The best sequence found before the wire snapped could be compared to a simple but effective version of the cobra undulations, see Fig. 6. The SAS makes S shapes by alternating between activating the top and right wires, with the top and left wires. This S shape is along the whole length of the SAS and so provides cobra-like undulations.

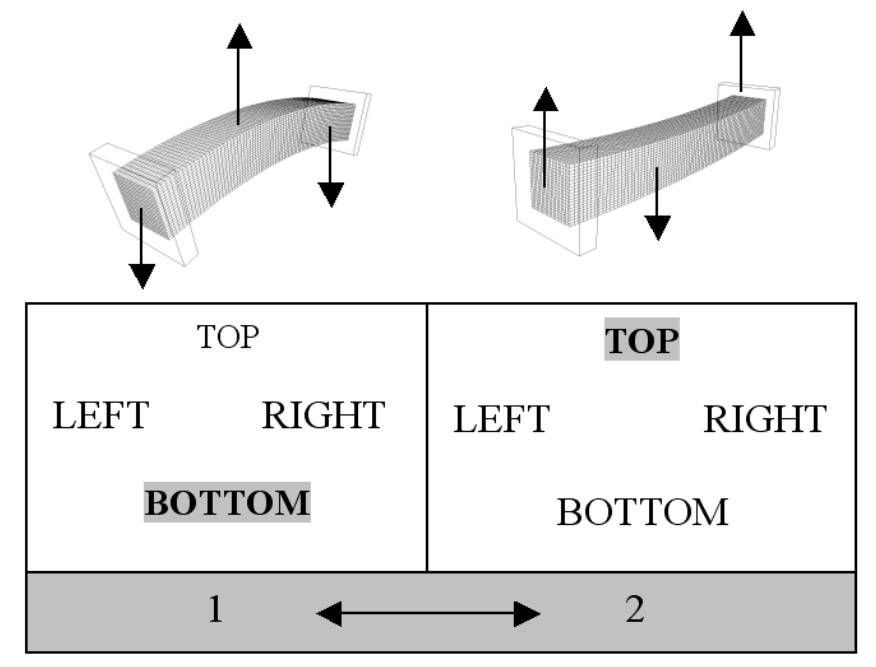

Fig. 7. Activation sequence of best sequence after damage resembles earthworm undulations.

After the SMA wire snapped, the GA eventually evolved a sequence that simply alternated between the top and bottom wires. Alternating between compression and extension means that the new method of locomotion that had been evolved much resembled the undulations of the earthworm, see Fig. 7. Though this did not propagate the SAS as efficiently as the sequences that had been found previously, this pattern of muscle activations recovered over $85 \%$ of the previous mobility when it only had $75 \%$ of its muscle intact.

\subsubsection{Summary of prototype snake robot investigation}

The results for this first robot have demonstrated the embodied evolutionary algorithm's ability to control a robot that uses SMAs as actuators. Despite having no knowledge of the morphology of the snake or its 
environment, the algorithm found an effective method of locomotion. During experimentation, one of the four SMA wires snapped, and by continuing to evolve the finite state machine that controlled which SMA wires were activated, the evolutionary algorithm adapted to the change and created a new method of locomotion which overcame the damage.

\subsection{The Second Self Adaptive Snake (SAS)}

Following this introduction to embodied control of the robot snake, further investigations were carried out to examine how the genetic algorithm could effectively recover the lost motion of the robot after damage. Calculating how best to recover from the damage of one of the actuators is clearly a non-trivial task. However, since the genetic algorithm is embodied in its environment, it should always be able to recover from damage effectively, without even being informed which SMA was damaged. In the following experiment, a new robot snake is constructed that has 12 actuators. We examine how the snake learns to move, though the translation between genotype and phenotype is much more complex, then some actuators are purposefully damaged to examine how the genetic algorithm can recover from this damage.

\subsubsection{Body}

The main bulk of the robot snake is made of foam. This provides a restoring force great enough to restore the wires to their original lengths after each activation. The SAS used in the second set of experiments uses twelve SMA wires (diameter $=0.176 \mathrm{~mm}$, activation (Austenite) temperature $=70^{\circ} \mathrm{C}$, recommended current $200 \mathrm{~mA}$ ). The body of the robot snake is split into four segments (this is an extension from the first SAS prototype described in section 3.1 which used only a single segment). These segments are readily detachable from each other and the whole structure can be expanded or segments replaced. Each segment has three SMA wires running down its length and a central copper wire that runs through the foam and supplies the power, much like a spinal chord carries nerve impulses to muscles through the body see Fig. 8. These four segments are connected together and the ends of the 'spinal chord' are connected together to create a continuous connection along the length of the robot snake see Fig. 9. The total weight of the robot snake is approximately $150 \mathrm{~g}$.

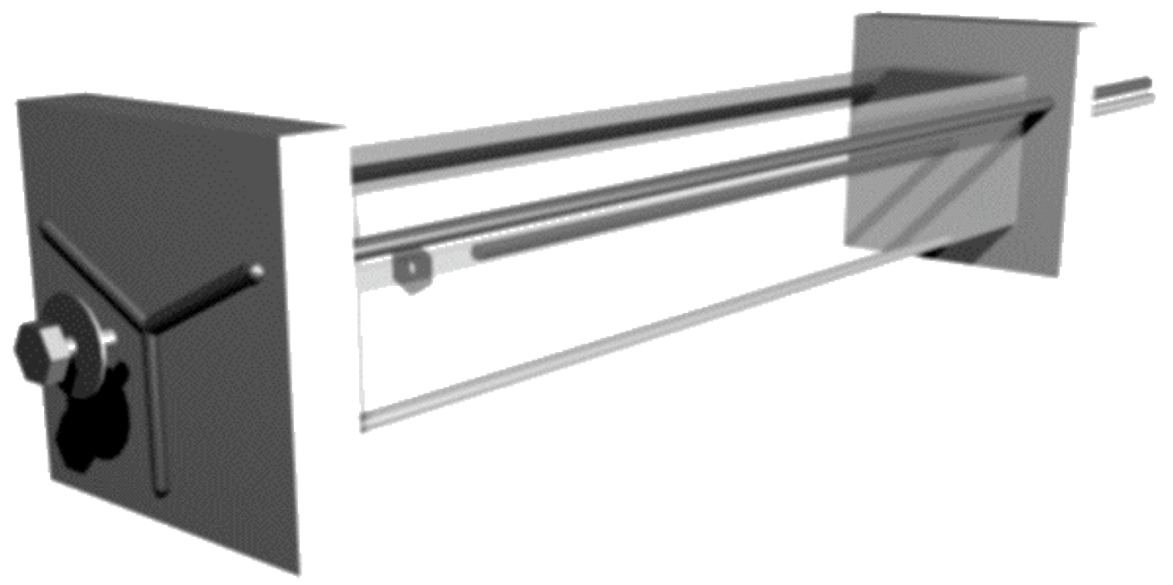

Fig.8. A single segment of the robot snake showing the three SMA wires and the central copper wire. 


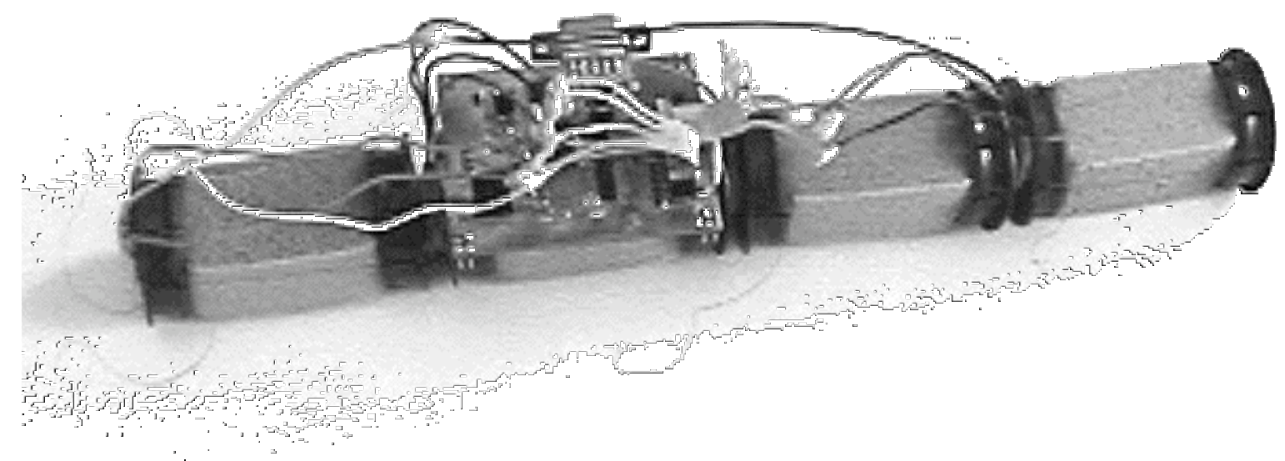

Fig. 9. A photograph of the SAS showing all four segments of the robot snake connected together.

\subsubsection{Finite State Machine (FSM)}

The SMA wire activations are determined by a FSM, this is evolved by a genetic algorithm designed especially for this task. The initial state of the FSM is constructed randomly and an array of FSMs was constructed representing a population of solutions.

As described in section 3.1.2, each member of the population is made up of a string of 0s and 1s. Each string consists of two segments, 'sequence' and 'next time'. For the second SAS, the 'sequence' length is twelve bits, and the 'next time' length is six bits, therefore the total length of each string is $2^{6} \times(12+6)=1152$ bits.

The program is allowed to send 30 sequences to the SAS before stopping, each lasting a total of approximately 40 seconds. Finite state machines that act as repeating patterns are easily created if the jump points point to each other in a loop of some sort. Indeed, very rarely is there a string that did not loop at all.

These patterns are then sent to the SAS for evaluation. The fitness given to each individual in the population is directly proportional to how far the SAS travels after the sequences have been sent. The same computer interfacing hardware as described in section 3.1.2 was employed. [11,12]

\subsubsection{Genetic Algorithm}

After the program has gone through the whole population, the genetic algorithm is used to evolve the next generation of solutions. In addition to the improved robot design, a more effective crossover operator was developed for the second SAS. This method is described below using a sample string containing 6 'sequence' bits. Two strings are chosen from the population using roulette wheel selection. See Fig. 10

\begin{tabular}{|l|l|l|l|l|l|l|}
\hline 101103 & $111000 \mathbf{4}$ & $101111 \mathbf{5}$ & $110000 \mathbf{1}$ & $101011 \mathbf{7}$ & $001010 \mathbf{3}$ & $111001 \mathbf{6}$ \\
\hline $010110 \mathbf{2}$ & $100100 \mathbf{4}$ & $100001 \mathbf{5}$ & $110111 \mathbf{3}$ & $001000 \mathbf{6}$ & $110010 \mathbf{2}$ & $100101 \mathbf{3}$ \\
\hline
\end{tabular}

Fig. 10. Example of two strings chosen from the population of current solutions.

The repeating pattern that each individual defines is illustrated below, see Fig. $11 \& 12$. These patterns can be extracted and better observed as simple loops. These simple loops are now taken as the chromosomes of the solutions and so only these parts of the complete string are crossed over. As can be observed in Figs 11 \& 12 , 
these chromosomes can be of varying lengths. In order to keep the length of the complete string constant, the shorter of the two chromosomes is taken, and a crossover section within its length is randomly cut. A section of the same size as that of the first chromosome is also cut at a random point along the second chromosome. These two sections are then swapped. Note that in order for the loops to remain functional, only the 'sequence' sections of the chromosomes are in fact crossed over leaving the 'next time' sections in unison, see Fig. 13.

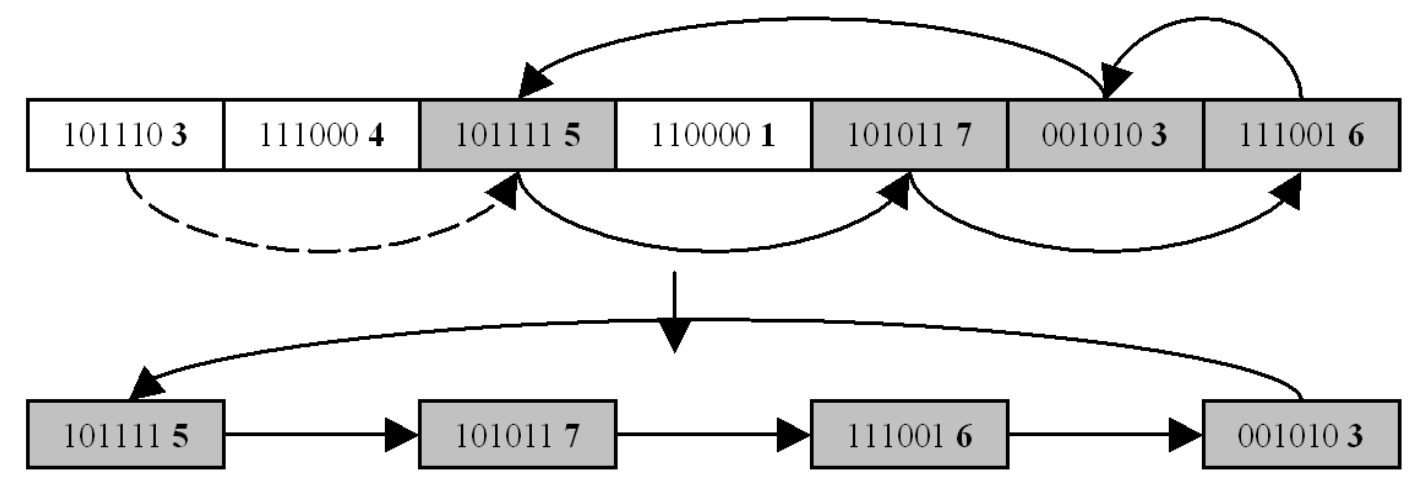

Fig. 11 The first string is converted into the loop that it represents.

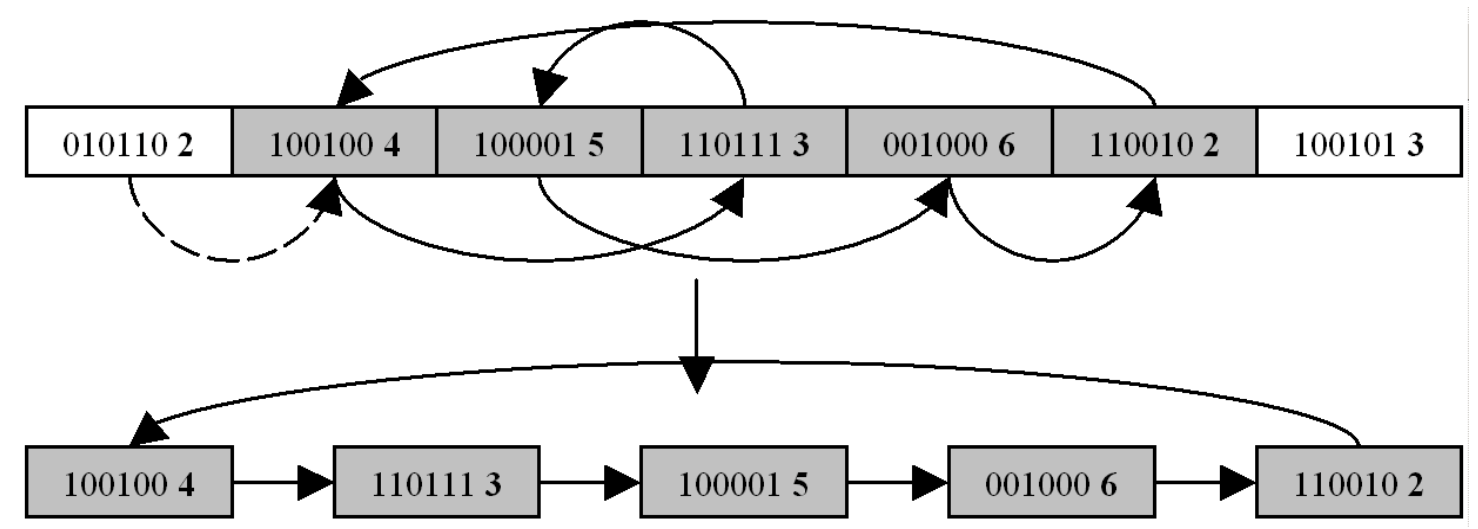

Fig. 12 The second string is converted into the loop that it represents.

Once this is done, mutation is carried out, where each bit of the string has an equal chance of being mutated, Fig. 14. These chromosomes are then placed back into their original slots in the complete string.

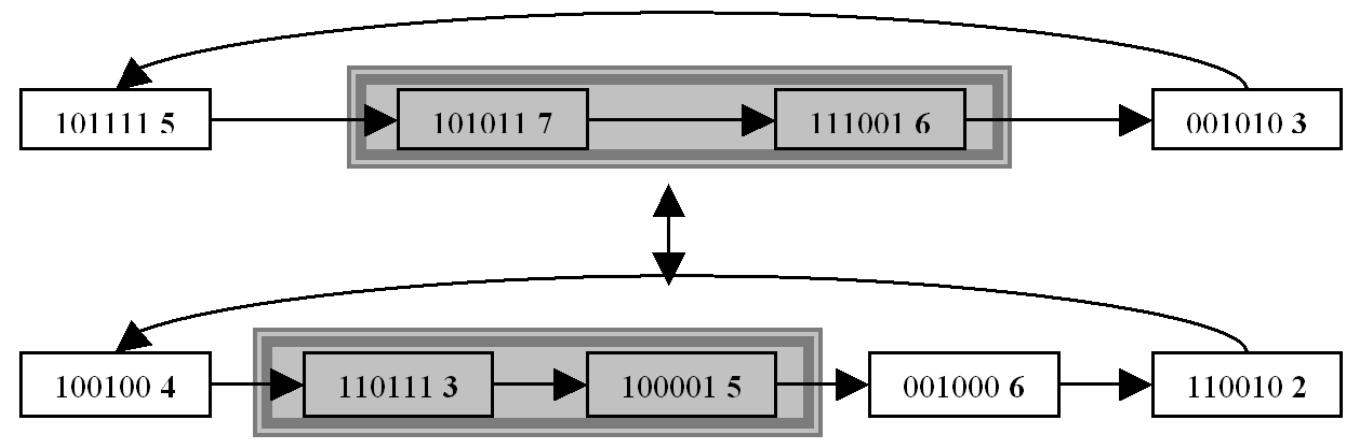

Fig. 13. Sections of the same size are swapped leaving the 'next time' sections intact. 

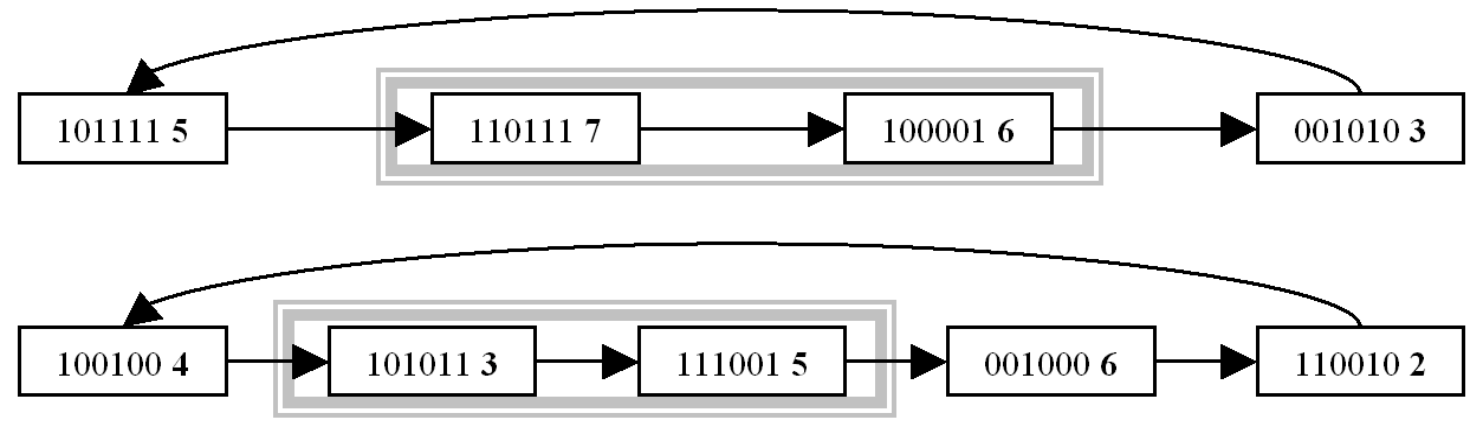

Fig. 14. Mutation is carried out only in the sections crossed over.

Only these chromosome fragments within the entire string are mutated, this ensures that these loops are accessed. If the rest of the sequence was mutated, then there is a good chance that a completely different loop would be accessed, thus the offspring would in no way resemble its parent and its fitness would be completely independent to that of the parent.

Though the sizes of both loops are conserved, mutation has a chance of completely redefining every loop, and so completely new solutions are still given a chance to be discovered which could have very different loop sizes. As described earlier, the genetic algorithm is elitist.

\subsubsection{Experimental Setup}

The objectives of this experiment are to investigate whether the genetic algorithm can find new methods of locomotion to recover from damage caused to the robot snake. In order to test this, a series of experiments were performed to investigate the evolution of movement for the SAS. The SAS's motion was evolved until the maximum fitness in the population of solutions remained unchanged for seven generations (chosen because of feasibility and time constraints). This was taken as a (somewhat local) maximum for the current configuration of the snake and its environment. Two wires that were widely used by the most fit individuals of the previous generations were then damaged. This was done by blocking access to them via software. The genetic algorithm was then allowed to continue evolving motion until another maximum was reached. Again two widely used SMA wires were damaged and evolution was again allowed to continue.

The microcontroller board was attached to the top of the robot snake and all the SMA wires were connected to the IO ports of the board. The only wires leaving the snake were the power supply and RS232 (both designed to minimise weight and resistance to movement). Another important factor that was realised in previous experiments $[11,12]$ and taken into consideration is that the resistance of the wires remains more or less constant no matter how far the SAS moves.

The distance travelled by the robot snake can be measured to the nearest millimetre. Experimentation was done to observe the true accuracy by which the distance travelled should be stated. The results show that the distance travelled was always within the nearest millimetre, and so the distances travelled by the SAS can be stated to the nearest millimetre without being over accurate [11].

As the program starts, an initial population of randomly generated finite state machines is created. Each individual finite state machine is then sent to the SAS for evaluation. The fitness of any member is determined solely on how far the snake travels (in $\mathrm{mm}$ ) in the forward direction. The total time taken to complete each generation is roughly twenty minutes (size of population (20)* time taken for each evaluation and reinitialisation $(\sim 1$ minute)). The total time taken for the whole experiment to complete was 33 hours. 


\subsubsection{Results}

Figure 15 shows the distance travelled at each generation. The greatest distance travelled by the SAS before any wires were damaged was $92 \mathrm{~mm}$. Immediately after two commonly used wires were damaged, the maximum distance travelled by the SAS in the next generation was $27 \mathrm{~mm}$. This is equivalent to a $71 \%$ drop in performance.

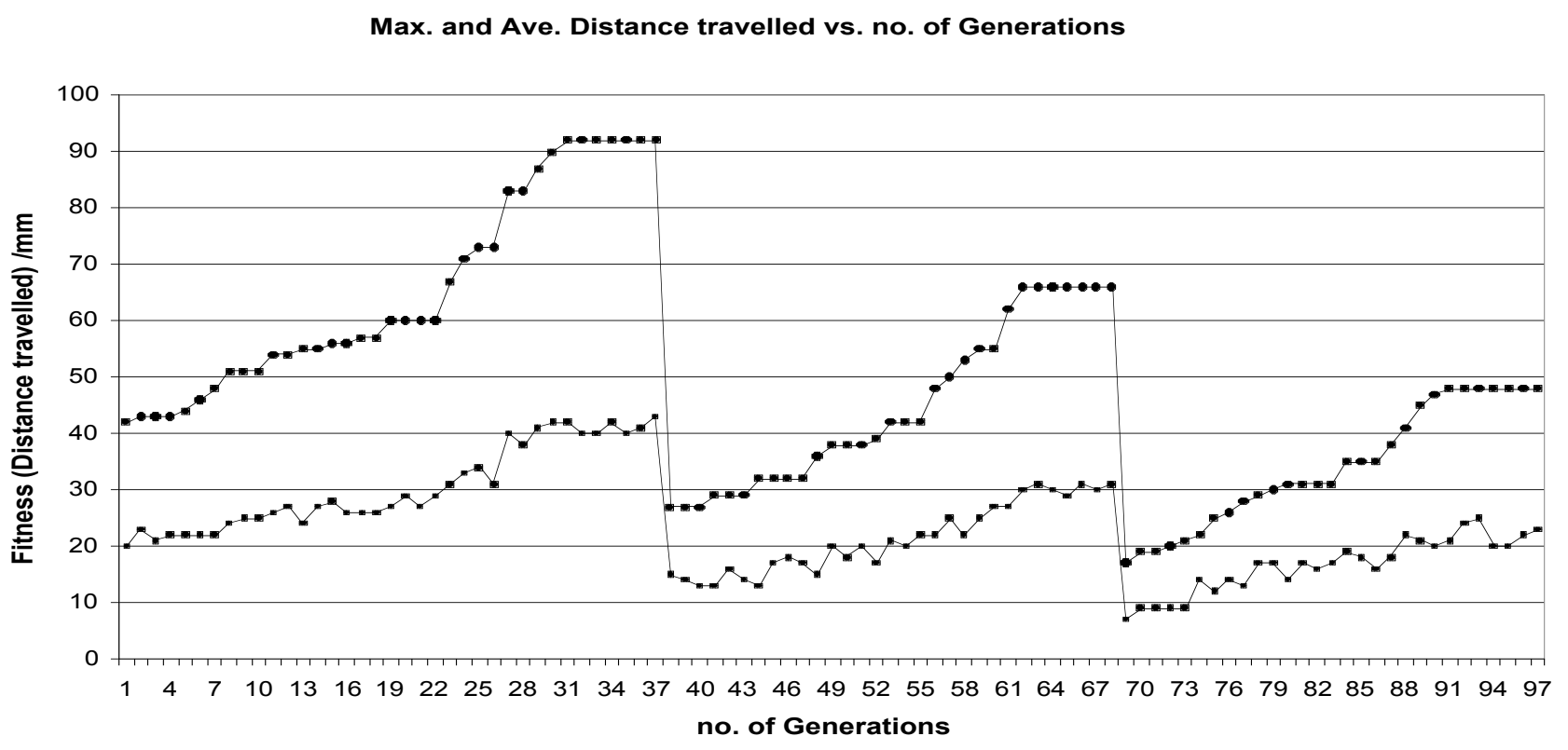

Fig. 15. Maximum fitness (bold) and average fitness plotted at each generation.

The SAS was then allowed to evolve for another 20 generations. This improved the distance travelled to a maximum of $66 \mathrm{~mm}$. This is an improvement in performance of $244 \%$, and motion has been recovered to within $72 \%$ of the original undamaged SAS. The SAS was then once again damaged, removing another two commonly used wires from action, resulting in a 74\% drop in performance. Finally the SAS was then allowed to evolve for a final 18 generations. This improved the distance travelled to a maximum of $48 \mathrm{~mm}$. This is an improvement in performance of $282 \%$, and motion has been recovered to within $52 \%$ of the original undamaged SAS.

\subsubsection{Analysis}

During the evolution of the SAS, numerous interesting methods of locomotion were carried out. This section seeks to analyse the control strategies of the solutions at the three maximums of the graph above. These methods of locomotion correspond to the best sequence found before any wires were damaged, the best sequence found after the first pair of wires were damaged, and the best sequence found after the second pair of wires were damaged.

Though the sequence lengths could vary from a length of one to a maximum of sixty-four, the sequence lengths that travelled the furthest seemed to average a length of ten. The evolved sequences at these points are very complex. By way of illustration, the two most commonly used wires out of twelve in those sequences are shown below, see Fig. 16, $17 \& 18$. In each of the sequences, the two wires highlighted were simultaneously activated and deactivated in nearly every step, while a complicated sequence of activations was sent to the rest of the wires. The two wires that are highlighted in Fig. 16 are also the wires that were subsequently damaged, resulting in a new sequence (and thus an entirely new movement plan) that relied on two different wires being activated and deactivated as illustrated in Fig.17. Once again, the two wires highlighted were the most commonly activated in this second solution and so were subsequently damaged resulting in the final solution. 
This relied on two different wires being activated and deactivated more often than any other wire in order to move effectively (and thus a third entirely new movement plan), as illustrated in Fig. 18.

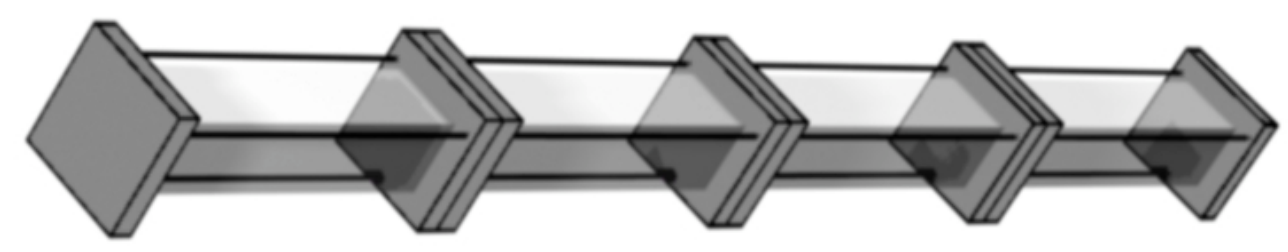

Fig. 16. Plan view of muscle wires in the four segments of the SAS (horizontal lines). The two most commonly used wires when there is no damage are shown in bold.

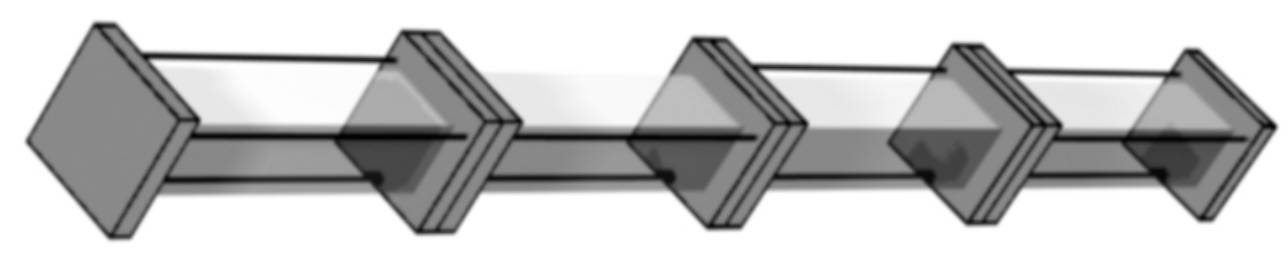

Fig. 17. The two most commonly used wires after two wires are damaged (bold), damaged wires are shown as dotted lines.

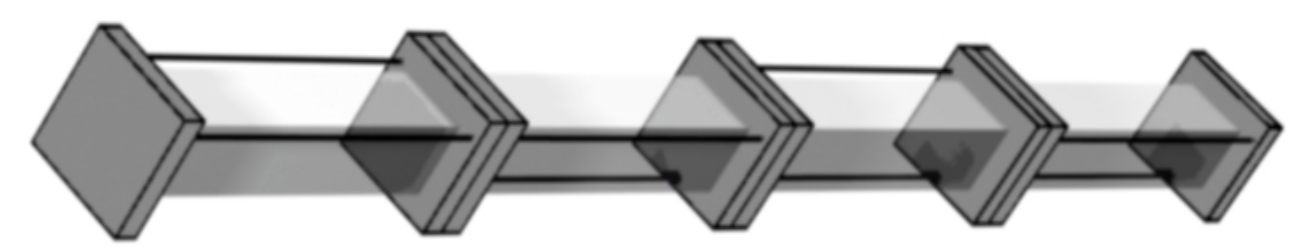

Fig. 18. The two most commonly used wires after four wires are damaged.

In previous experiments performed with fewer wires, the motion exhibited by the SAS could be compared to various types of snake undulation $[11,12]$. This has not been the case with twelve wires. There are two possible reasons for this. Firstly that the genetic algorithm had not had enough time to evolve elegant snakelike motion. The second reason could be that the genetic algorithm was exploiting asymmetries in the construction of the robot snake, the difference in the tautness of the SMA wires, and the individual properties of these wires. This being the case, these more asymmetrical sequences would prove to be more effective for creating motion than if the SAS had explicitly tried to mimic snakelike motion.

\subsection{Summary of second snake robot investigation}

This section has shown that it is possible to use a combination of a self-adaptive snake robot (using shape memory alloys to provide flexibility) and embodied evolutionary control to produce a robot that is capable of recovering from damage by learning new movement strategies. The experiments demonstrated that a high percentage of locomotion can be recovered more than once, even when muscle wires crucial to a locomotion strategy are disabled.

\section{The Adaptive Antenna}

Having explored damage recovery in a novel roving robot, the power of embodied robot control is further investigated, this time with an antenna constructed using SMAs as actuators. The SMAs contort of the surface 
of the antenna allowing it to alter its reception capabilties. The following experiment will investigate whether the embodied evolutionary algorithm is able to control SMA activation and obtain the best reception in a noisy and unpredictable environment.

The receiver antenna created in this work was designed to make best use of the 16 SMA wires that were used to manipulate it. The antenna is designed in the shape of an umbrella, see Fig. 19. The edges of the dish have 16 SMA wires attached to them. These SMA wires are attached to the base and then connected to the circuit board that can power them individually. As described, the SMA wires used in this work simply contract by $\sim 5-8 \%$. This means that though the antenna would have 16 degrees of freedom (resulting in over 65000 different orientations), these would never drastically affect the overall shape of the antenna. This is useful for the genetic algorithm used to control the wires, as a change in one of the wires (and corresponding bit in the genome) would not result in a drastically different configuration - good for evolvability. The activation of the 16 SMA wires has the result of bending and contorting the surface of the curved surface of the antenna.

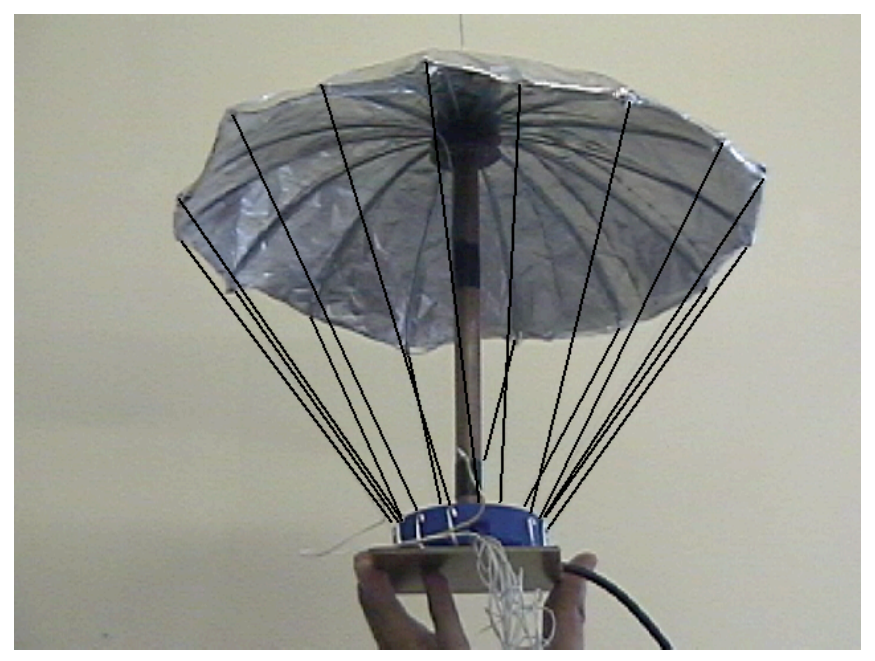

Figure 19 A photo of the adaptive antenna (SMA wires highlighted in image).

The measurements and features of the antenna are illustrated in Fig. 20 below.
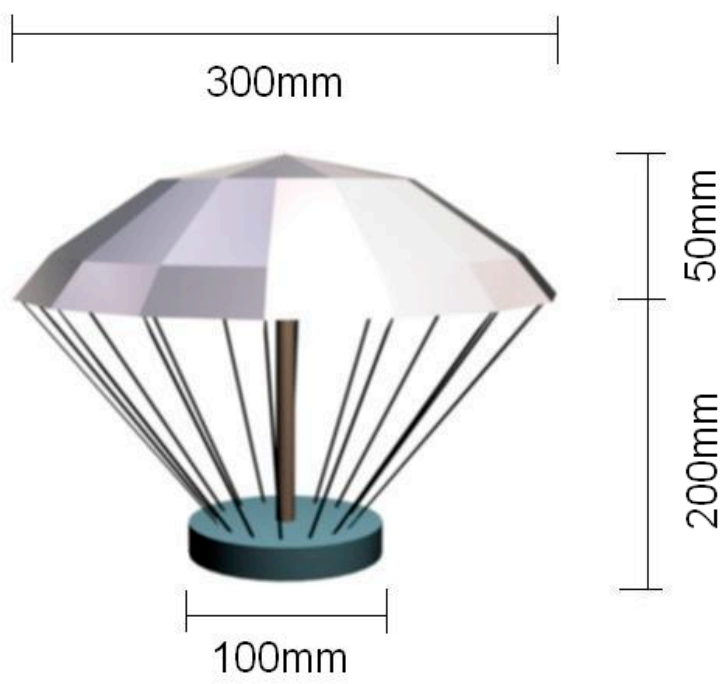

Figure 20 The dimensions of the adaptive antenna with 16 SMA wires attached. 


\section{The Genetic Algorithm}

\subsection{The chromosome structure}

There are a total of 16 SMA wires that are available for activation by the genetic algorithm. Each individual in the population of solutions is described by a 16 bit string of ones and zeros. A one in the string would mean an active SMA wire. In the example below, there are seven SMA wires activated, see Fig. 21.

\begin{tabular}{|l|l|l|l|l|l|l|l|l|l|l|l|l|l|l|l|}
$\mathbf{0}$ & $\mathbf{1}$ & $\mathbf{1}$ & $\mathbf{0}$ & $\mathbf{1}$ & $\mathbf{0}$ & $\mathbf{0}$ & $\mathbf{1}$ & $\mathbf{0}$ & $\mathbf{1}$ & $\mathbf{0}$ & $\mathbf{1}$ & $\mathbf{0}$ & $\mathbf{0}$ & $\mathbf{0}$ & $\mathbf{1}$ \\
\hline
\end{tabular}

Figure 21 An individual is defined by a 16 bit string.

\subsection{Genetic Operators}

The crossover operator takes $n$ bits from two chromosomes and swaps them. In the following example, two 16 bit strings are taken and 4 bits are selected from each of them, see Fig. 22.

\begin{tabular}{|l|l|l|l|l|l|l|l|l|l|l|l|l|l|l|l|}
\hline $\mathbf{0}$ & $\mathbf{1}$ & $\mathbf{1}$ & $\mathbf{0}$ & $\mathbf{1}$ & $\mathbf{0}$ & $\mathbf{0}$ & $\mathbf{1}$ & $\mathbf{0}$ & $\mathbf{1}$ & $\mathbf{0}$ & $\mathbf{1}$ & $\mathbf{0}$ & $\mathbf{0}$ & $\mathbf{0}$ & $\mathbf{1}$ \\
\hline
\end{tabular}

\begin{tabular}{|l|l|l|l|l|l|l|l|l|l|l|l|l|l|l|l|}
\hline $\mathbf{1}$ & $\mathbf{1}$ & $\mathbf{0}$ & $\mathbf{0}$ & $\mathbf{1}$ & $\mathbf{1}$ & $\mathbf{1}$ & $\mathbf{0}$ & $\mathbf{0}$ & $\mathbf{1}$ & $\mathbf{1}$ & $\mathbf{1}$ & $\mathbf{0}$ & $\mathbf{1}$ & $\mathbf{1}$ & $\mathbf{0}$ \\
\hline
\end{tabular}

Figure 22 Four bits are selected from two 'parent' individuals.

These bits are then swapped and the resulting individuals are now ready for mutation, see Fig. 23.

\begin{tabular}{|l|l|l|l|l|l|l|l|l|l|l|l|l|l|l|l|}
\hline $\mathbf{0}$ & $\mathbf{1}$ & $\mathbf{0}$ & $\mathbf{0}$ & $\mathbf{1}$ & $\mathbf{1}$ & $\mathbf{0}$ & $\mathbf{1}$ & $\mathbf{0}$ & $\mathbf{1}$ & $\mathbf{1}$ & $\mathbf{1}$ & $\mathbf{0}$ & $\mathbf{0}$ & $\mathbf{0}$ & $\mathbf{1}$ \\
\hline
\end{tabular}

\begin{tabular}{|l|l|l|l|l|l|l|l|l|l|l|l|l|l|l|l|}
\hline $\mathbf{1}$ & $\mathbf{1}$ & $\mathbf{1}$ & $\mathbf{0}$ & $\mathbf{1}$ & $\mathbf{0}$ & $\mathbf{1}$ & $\mathbf{0}$ & $\mathbf{0}$ & $\mathbf{1}$ & $\mathbf{0}$ & $\mathbf{1}$ & $\mathbf{0}$ & $\mathbf{1}$ & $\mathbf{1}$ & $\mathbf{0}$ \\
\hline
\end{tabular}

Figure 23 The bits are swapped, resulting in two new 'child' individuals.

Mutation is also applied occasionally. This involves randomly choosing $m$ bits from an individual and flipping them (ones becoming zeros and vice versa).

\subsection{Selection and Initialisation}

Elitism was used in the genetic algorithm, using roulette wheel selection. The initial population is created randomly. Each bit of each chromosome had a 0.3 chance of being a one (activated SMA wire).

\subsection{Fitness function}

As will be described in the next section, the fitness of each individual was not the absolute number of strings received by the antenna during that individual's particular SMA wire activations, but instead the relative number of received "PING" strings when compared to the neutral state at that time. The fitness function is as follows:

\section{Fitness $=$ activated_reading - background_reading $+C$}

Where activated_reading is the number of strings successfully received while the antenna is reshaped according to the current individual design, background_reading is the number of strings successfully received when the antenna is in its relaxed, default shape (measured just before activated_reading is taken) and $C$ is a constant that ensures that the fitness is always positive. Therefore if the value of Fitness is less than $C$, the SMA activated individual has performed worse than if the wires were not activated in the first place. Likewise, if the value of Fitness is greater than $C$, the SMA activated interval has performed better. 


\subsection{Experimental Setup}

To assess the reception capabilities of the adaptive antenna in a noisy environment, a transmitter was constructed that transmitted the string "PING" twelve times a second at a frequency of $433 \mathrm{MHz}$. The receiver antenna was connected to a PIC microcontroller board that attempted to receive the "PING" strings. If the whole string was received uncorrupted by noise, then that would be considered a successful transmission. Any corrupted string received (e.g. "PONG") would be rejected.

When the transmitter is placed within a metre of the antenna, every transmitted "PING" string is received. However as the transmitter is moved further away from the receiver antenna, the number of strings successfully transmitted reduces until eventually not even a single transmitted string is ever received by the antenna.

The experimentation was done within a lab at University College London with many unpredictable sources of noise. (These were caused by computers, the nearby BT telecommunication tower, mobile phones and other equipment in normal use.) The transmitter was placed just outside the room in order to reduce the signal strength and make the noise within the room more problematic, see Fig. 24.

These sources of noise, along with others that were not identifiable, did not have a constant and continuous effect on the receiver. This meant that the noise levels varied the whole time. The transmitter was set up in such a way so that the receiver could receive $70 \%$ or less of the strings sent. This was done to ensure that the noise present within the room had a significant enough impact on the receiver so as to corrupt some of the "PING" strings being transmitted.

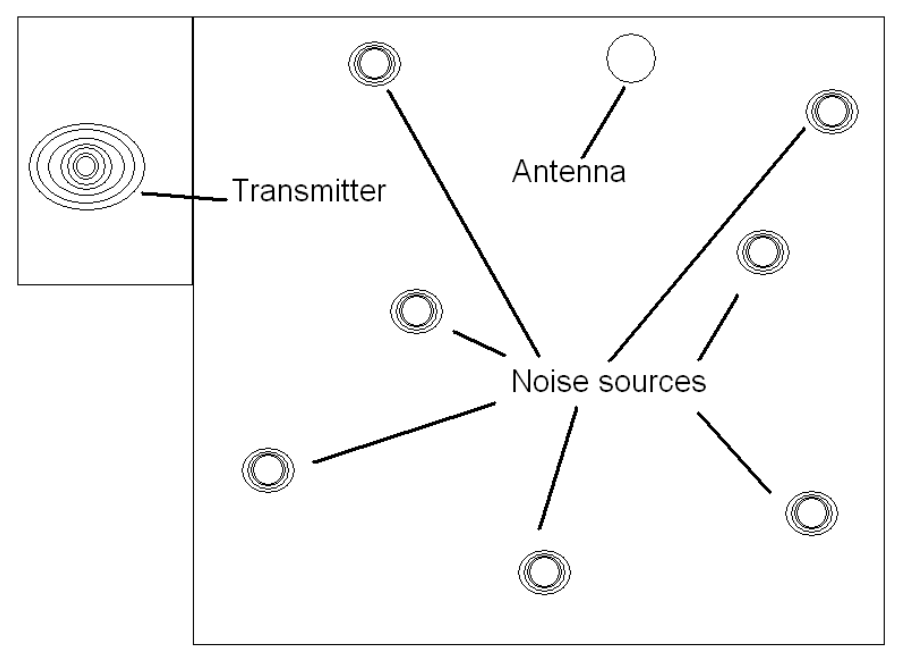

Figure 24 A plan of the room.

Figure 25 illustrates the noise during part of one run. In ideal conditions, the transmitted string can be received up to 27 times correctly in each test. The dark line shows (with the antenna in its default shape) just how noisy the environment is. The paler line shows the effect of activating the antenna into a specific shape, within a split second of each previous test. Clearly, activating the SMA wires do affect reception. Experiments have shown that the wires themselves do not seem to be receiving or interfering with reception - only the change of antenna shape that they cause seems to affect reception. (Interestingly, earlier systems evolved to deactivate all wires, for the power drain caused by their activation reduced the power to the receiver electronics. The version described here now uses a separate power source for the wires.) 


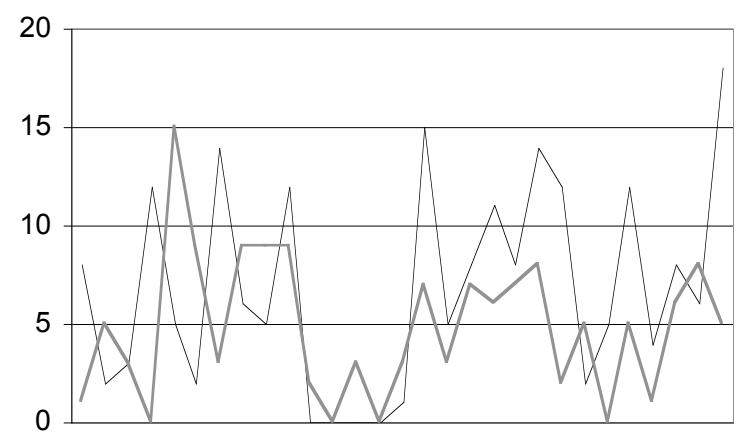

Figure 25 Typical equilibrium (dark line) and activated (light line) reception levels over time.

As described in the previous section, the fitness of an individual is measured relative to the performance of the relaxed antenna. Both are measured immediately one after the other in an attempt to ensure they are receiving signals under the same noise conditions. So the actual fitness of any individual is actually the difference between the two measurements.Figure 26 illustrates the fitness of the individual shown in figure 8 under different noise conditions. The value of $C$ is 27 , meaning that if the fitness is below the dashed line, the individual performs worse than the neutral antenna. If above the dashed line, it performs better.

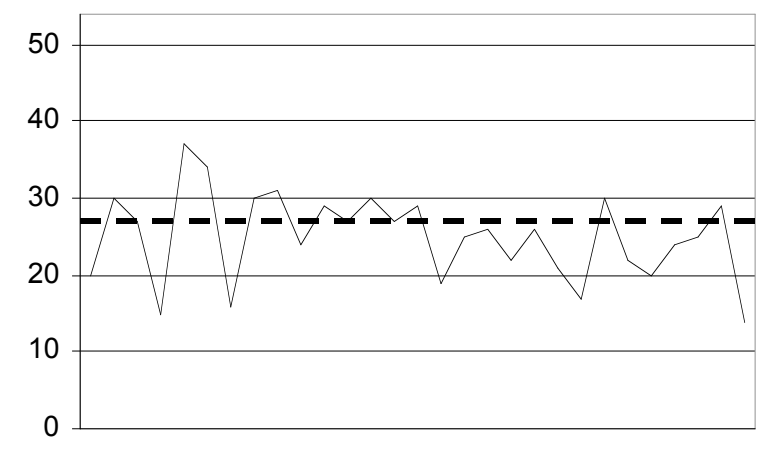

Figure 26 Signal reception quality relative to the background noise.

The experiment was run several times. The longest recorded run lasted approximately 12 hours, and the results for this run are reported here (although other runs showed similar results). Each individual was given 2 seconds to receive as many of the "PING" strings as possible in both the neutral and configured states. The genetic algorithm parameters are as follows:

- Population size: 20 individuals

- Crossover: 4/16 bits crossed, each time.

- Mutation rate: Every fifth generation, there is a $1 / 16$ chance of one bit mutating.

- Generations: 300.

- $\quad$ The value of $C=27$.

\subsection{Results}


Figure 27 shows the results of the experiment. Although subtle because of the extensive noise, it is clear that the population average increases above the value of $C$ as evolution progresses. It also shows that the maximum fitness remains at around the same level, but significantly the amplitude of the noise seems to be lower. Finally, the chart shows clearly how the minimum fitness increases - demonstrating evolution of antenna configurations that minimise the harmful effects of the noise.

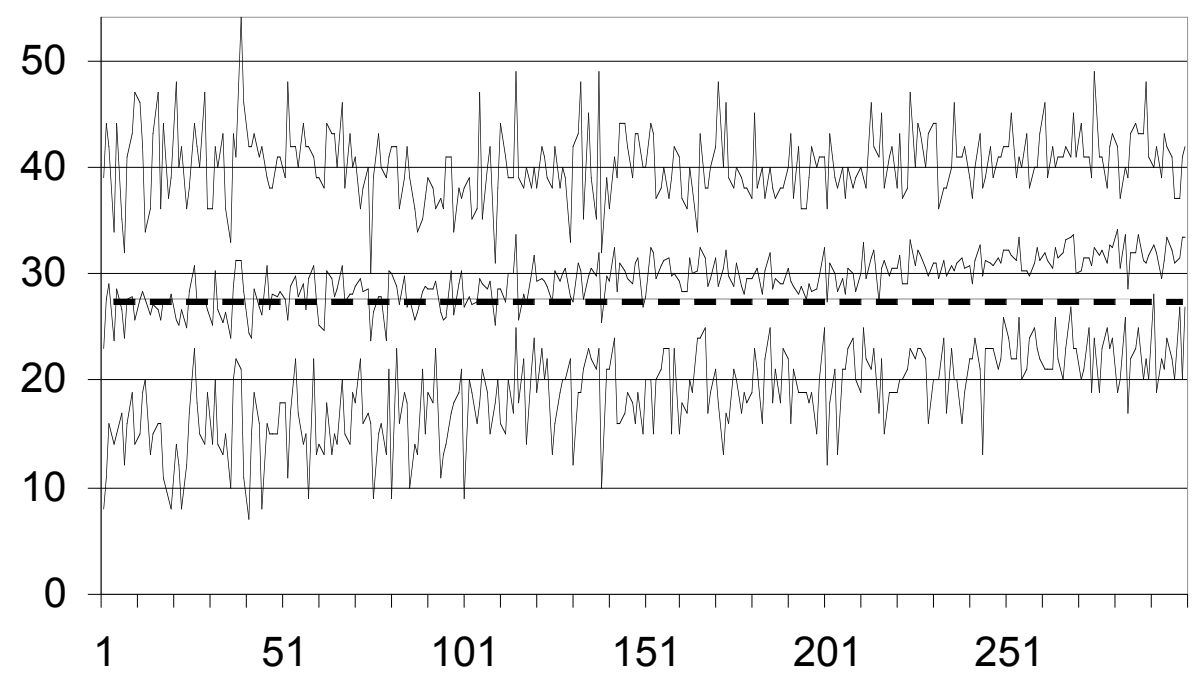

Figure 27 Fitnesses of evolving antenna designs per generation. Top line shows population best, middle line shows population average, bottom line shows population worst. The dashed line at a value of 27 is where the activated configuration receives the same number of strings as in the neutral state.

\subsection{Analysis}

The final population contained mostly similar genomes. Figure 28 shows the "average genome" (formed by choosing the most commonly appearing $1 \mathrm{~s}$ and $0 \mathrm{~s}$ at each genome position in the population). Figure 29 shows how this genome translates into an antenna design.

\begin{tabular}{|l|l|l|l|l|l|l|l|l|l|l|l|l|l|l|l|}
\hline $\mathbf{1}$ & $\mathbf{0}$ & $\mathbf{0}$ & $\mathbf{0}$ & $\mathbf{0}$ & $\mathbf{0}$ & $\mathbf{1}$ & $\mathbf{1}$ & $\mathbf{1}$ & $\mathbf{1}$ & $\mathbf{1}$ & $\mathbf{1}$ & $\mathbf{1}$ & $\mathbf{0}$ & $\mathbf{0}$ & $\mathbf{1}$ \\
\hline
\end{tabular}

Figure 28 "Average genome" in the final population. 


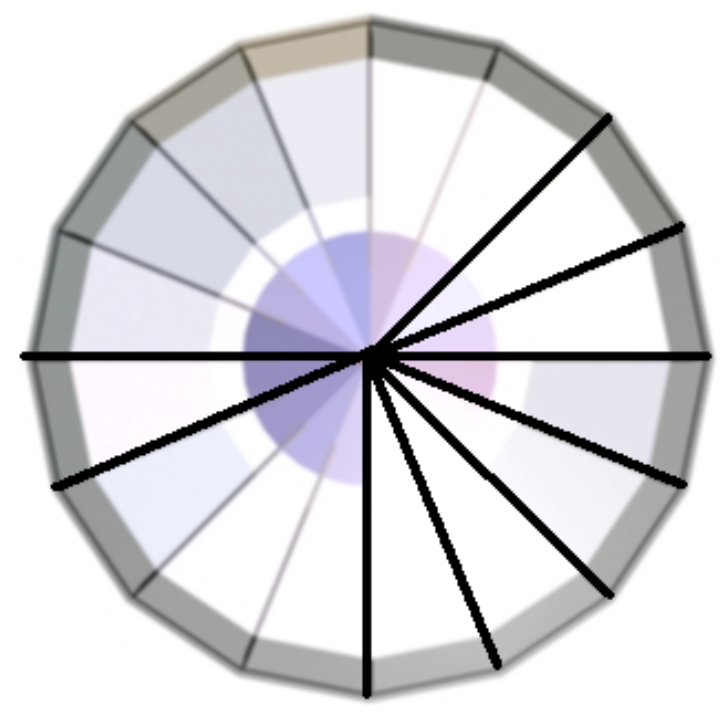

Figure 29 The top view of the antenna with the activated SMA wires indicated. The transmitter is located directly to the left of this.

To obtain some further measure of the quality of this final, evolved solution, the configured antenna was compared to the antenna in its relaxed state in 20 tests (during which the background noise varied as always). Figure 30 shows how the evolved antenna maintains a high reception rate, compared to the neutral configuration, which shows highly variable reception caused by noise. Figure 31 shows the normalised results or fitness during this time.

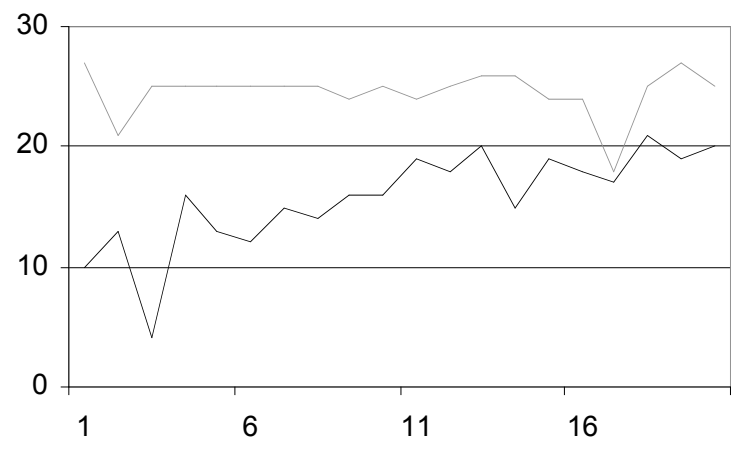

Figure 30 The activated antenna (light line) compared to the neutral state reception (dark line). 


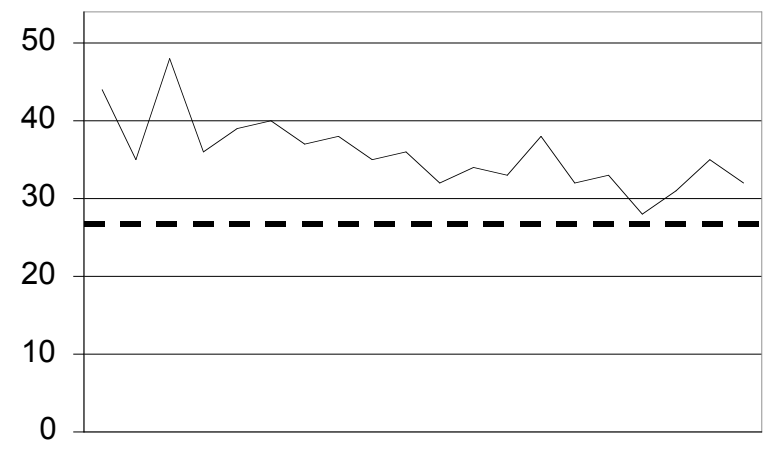

Figure 31 The normalised graph of the activated antenna's reception.

Significantly, the evolutionary run that created this design was performed overnight. It was hypothesised that the noise conditions at night would greatly differ from those present during the day. Therefore the same genome was again compared to reception of the neutral state during the daytime. The results are plotted in Fig. 32 and 33.

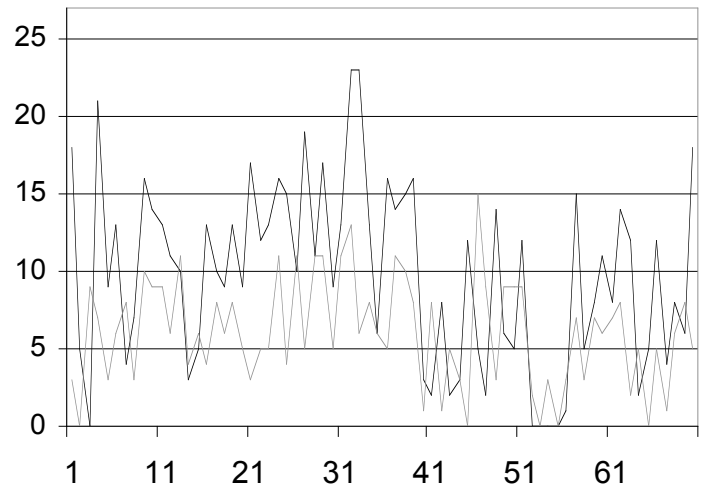

Figure 32 The neutral state reception (dark line) and the reception of the activated antenna (light line).

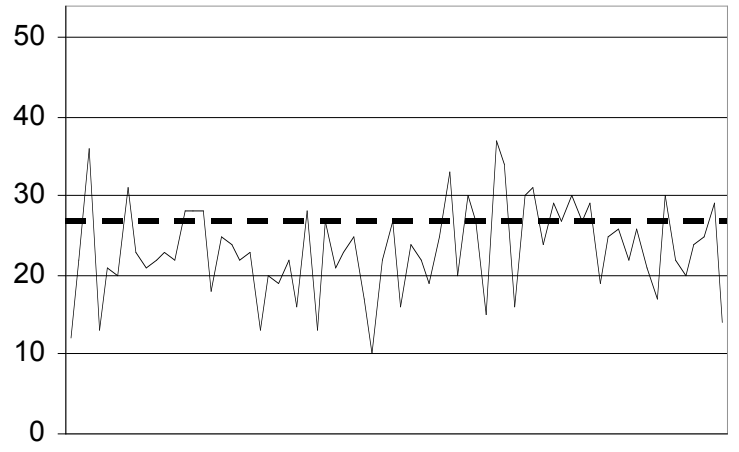

Figure 33 Normalised reception of activated antenna. 
The activated antenna now shows a completely different result - it consistently underperforms. This means that although it has adapted well to the main sources of noise during the night, during the day, when different noise sources were present, this configuration is now a maladaptation that harms performance. Of course, while this might be a problem for traditional, fixed antennas, in this system the configuration can simply be re-evolved for daytime noise (or for noise during the day and night).

\subsection{Summary of adaptive antenna investigation}

This section investigated whether an antenna, whose configuration was controlled by the activation of SMA wires, could adapt its shape in order to maximise its reception of a transmitted signal. This was done through embodied evolution by a genetic algorithm.

The GA discovered a noise tolerant antenna that minimised the effect of noise on the system. A more intensive investigation was then carried out, which reconfirmed this solution's tolerance to noise. The same system was then tested under very different noise characteristics. It was observed that adaptation to the previous conditions was so precise that in the vastly different noise environment, the solution was no longer ideal and in fact performed consistently worse then the neutral state orientation.

\section{Discussion}

Conventional robot control techniques rely heavily on careful analysis of the robot and the anticipated environment of the robot. Typically, computer simulation is employed to model robot and environment, before the control software is integrated with the hardware and deployed. It is becoming increasing clear that such approaches are flawed when the detailed characteristics of the robot may be unknowable (or may become unknowable through damage) and when the environment of the robot may be unknown (or may become unknown through environmental change). In this work we have illustrated two such situations. First, a robot which uses shape memory alloys as actuators was deployed on a surface and required to propel itself along that surface. Detailed modelling of such a robot is impossible, for the complex interactions of shape memory alloy with the structure of the robot and an unknown surface are not understood well enough. Second, an adaptive antenna was deployed in a noisy environment and required to maximise its own reception. Again, modelling of the device was impossible, and its environment was sufficiently complex that it was effectively entirely unpredictable.

A solution to the problem of robot control under these circumstances was identified and demonstrated in this work: the use of embodied evolutionary algorithms for robot control. The key feature of the approach is that no simulations are employed. Instead, each evolving control program is uploaded into the actual physical device in its real environment, and performance assessed "in the field". True fitness measures of candidate solutions are then used by the evolutionary algorithm to create control programs designed to exploit the actual properties of robot and environment at the time they are occurring. This on-the-spot adaptation is a true example of embodiment - the robot/antenna affects its environment by moving in that environment, while the environment plays an immediate and important role in affecting the device and its performance, and thus affecting the subsequent control of the device in the immediate future. It is not necessary for a robot control program to "know" the status of the robot or of the environment - all that is necessary is that the robot be flexible enough to adapt, and some measurable performance metric can be obtained. An evolutionary algorithm is then able to exploit whatever exists and is controllable in order to make the device work. 


\section{Conclusions}

This paper sought to investigate the effectiveness of an embodied evolutionary algorithm in the control of two physical robots: a self-adaptive snake robot and an adaptive antenna. It is evident from the results that the embodied evolutionary algorithm gives the robots an innate adaptability. The embodied evolutionary algorithm was able to effectively control both robot snakes as well as recover from damage to the actuators. The same algorithm was then able to contort the surface of an antenna so as to reduce the affect of noise on it, thus maximising its ability to receive data.

\section{References}

1. Bentley, P. J. (Ed.) (1999) EvolutionaryDesign by Computers. Morgan Kaufmann Pub.

2. Bentley, P. J. and Corne, D. W. (Ed.) (2002) Creative Evolutionary Systems. Morgan Kaufmann Pub.

3. Bererton, $\mathrm{C}$ and Khosla, P. A Team of Robots with Reconfiguration and Repair Capabilities. In proceedings International Conference on Robotics and Automation, 2000.

4. Bird, J. and Layzell, P. (2002) The Evolved Radio and its Implications for Modelling the Evolution of Novel Sensors. In Proc. of Congress on Evolutionary Computation (CEC2002).pp1836-1841.

5. Brooks, R. (1992) Artificial life and real robots. Proc. First European Conference on Artificial Life.

6. Dittrich, P., Skusa, A., Kantschik, W., and Banzhaf, W. (1999) Dynamical Properties of the Fitness Landscape of a GP Controlled Random Morphology Robot. Proc. of GECCO '99, p. 1002-1008, Morgan Kaufmann, San Francisco, CA, 1999

7. Elliott, R. S. (2002) Antenna Theory \& Design (IEEE Press Series on Electromagnetic Wave Theory) John Wiley and Sons Inc.

8. Hanna, S and Haroun Mahdavi, S. (2004) Creativity Through Embodiment: A computational model exemplified by microstructure design. Sent to Computational and Cognitive Models of Creative Design (HI 2004)

9. Haroun Mahdavi, S. (2002) Evolving Motion Master's Dissertation, MSc IS, University College London, Dept. Computer Science..

10. Haroun Mahdavi, S. and Bentley, P. J.(2003) Evolving Motion of Robots with Muscles. In Proc. of EvoROB2003, the 2nd European Workshop on Evolutionary Robotics, EuroGP 2003. pp. 655-664.

11. Haroun Mahdavi, S. and Bentley, P. J. (2003) An Evolutionary Approach to Damage Recovery of Robot Motion with Muscles. Proc. of the European Conference on Artificial Life (ECAL 2003).

12. Haroun Mahdavi, S. and Bentley, P. J. (2003) Evolving Noise Tolerant Antenna Configurations Using Shape Memory Alloys. Proc. of Computational Intelligence, Robotics and Autonomous Systems (CIRAS 2003)

13. Hodgson, Darel E. (2002), Shape Memory Applications, Inc., Ming H. Wu, Memory Technologies, and Robert J. Biermann, Harrison Alloys, Inc. www.sma.com/Shape Memory Alloys.htm

14. Hornby, Gregory S. and Pollack, Jordan. B. (2001). Evolving L-Systems To Generate Virtual Creatures. Computers and Graphics, 25:6, pp 1041-1048.

15. Huntsberger, T and Aghazarian, H. (2004) Learning to Behave: Adaptive Behavior for Planetary Surface Rovers. Proc. of Simulationi of Adaptive Behavior (SAB 04)

16. Husbands, Phil and Meyer, Jean-Arcady (Eds.) (1998) Evolutionary Robotics, First European Workshop, EvoRbot98, Paris, France, April 16-17, 1998, Proceedings. Lecture Notes in Computer Science 1468 Springer 1998, ISBN 3-540-64957-3

17. Husband P., Smith T., Jakobi N. \& O'Schea M. (1999). Better living through chemistry: Evolving GasNets for robot control. Connection Science, (10)3-4:185-210.

18. Jakobi, N. (1997), 'Evolutionary robotics and the radical envelope of noise hypothesis', Journal Of Adaptive Behaviour 6(2), 325-368

19. Kamimura, A et al. (2001) Self-Reconfigurable Modular Robot - Experiments on Reconfiguration and Locomotion. Proc. of IEEE Int. Conf. on Intelligent Robots and Systems, 590-597, Hawaii, USA. 
20. Kárník, L. (1999) The possible use of artificial muscled in biorobotic mechanism. In ROBTEP'99, Kosice, SF TU Kosice, 1999, pp. 109-114. ISBN 80-7099-453-3.

21. Linden, D. S. (1997). Automated Design and Optimisation of Wire Antennas Using Genetic Algorithms. Ph.D. dissertation, MIT.

22. Lohn, J.D. Kraus, W.F. Colombano, S.P. Evolutionary Optimization of Yagi-Uda Antennas, Proc. of the Fourth International Conference on Evolvable Systems, Tokyo, October 3-5, 2001, pp. 236-243.

23. Lohn, J.D. Kraus, W.F. Linden, D.S. Evolutionary Optimization of a Quadrifilar Helical Antenna, Proc. of the IEEE AP-S International Symposium and USNC/URSI National Radio Science Meeting, June, 2002, to appear.

16. Mataric, M. and Cliff, D (1996) Challenges in evolving controllers for physical robots. Robots and Autonomous Systems 67-83

24. MC68HC908JK1 Microcontroller User's Manual (2002). Motorola Literature Distribution: P.O. Box 5405, Denver, Colorado 80217

25. Meyer J, Husbands P and Harvey I (1998) Evolutionary Robotics: A Survey of Applications and Problems. EvoRobots 1998: 1-21

26. Michael, J. Robodyne Cybernetics Ltd. www.fractal-robots.com

27. Mihalachi, D. and Munerato, F. (1999) Snake-like mobile micro-robot based on 3 DOF parallel mechanism. In Proc. of PKM'99 MILAN November 30, 1999.

28. Mihalachi, D. (2000) Systeme de Commande Temps Reel Distribue pour un Mini Robot Autonome de type Serpent. Doctorat de L'Universite de Metz, These Présentée à L’U.F.R. Mathematique, Informatique, Mecanique, Automatique.

29. Mills, Jonathan W. (1999) Stiquito for beginners. ISBN 0-8186-7514-4, 1999.

30. Quick, T: 1999, The essence of embodiment: a framework for understanding structural coupling between system and environment, Proc Third International Conference on Computing Anticipatory Systems, (CASYS,'99).

31. Støy, K (2002) Using Role Based Control to Produce Locomotion in Chain-Type Self-Reconfigurable Robots. IEEE Transactions on Mechatronics, 7(4), pages 410-417, 2002. 